\title{
FESetup: Automating Setup for Alchemical Free Energy Simulations
}

\author{
Hannes H Loeffler ${ }^{1, *,+}$, Julien Michel ${ }^{2,+}$, Christopher Woods ${ }^{3, \not}$ \\ ${ }^{1}$ Scientific Computing Department, STFC Daresbury, Keckwick Lane, Warrington WA4 4AD, UK; \\ E-mail: Hannes.Loeffler@stfc.ac.uk \\ ${ }^{2}$ EaStCHEM School of Chemistry, University of Edinburgh, West Mains Road, Edinburgh EH9 3JJ, \\ $\mathrm{UK}$ \\ ${ }^{3}$ BrisSynBio, University of Bristol, 8-10 Berkeley Square, Bristol BS8 1HH, UK
}

\section{FESetup Usage}

The source code repository including a Wiki, tutorials, a manual page, a discussion board, bug tracker and feature tracker are hosted through $\mathrm{CCP}-$ Forge on http://ccpforge.cse.rl.ac.uk/gf/project/ccpbiosim/. The following will give a brief overview how to use FESetup. More details can be found in the tutorials and the manual page on our web site.

\section{Input examples}

The following simple example (typed into a file e.g. test.in) sets up all required input files for computation of the solvation free energy of a set of small organic molecules. Force fields, MD engine and a log file are set in the [globals] section (the keywords should be self-explaining). The [ligand] section will create a rectangular box with 10 Å padding and neutralize the simulation system. Vacuum setup will always be created. Each named molecule will be parametrized through GAFF and AM1-BCC. Input structures are searched for in a sub directory smallmols. The molecule names are directories within and contain the actual structure files, a PDB file in this case. 


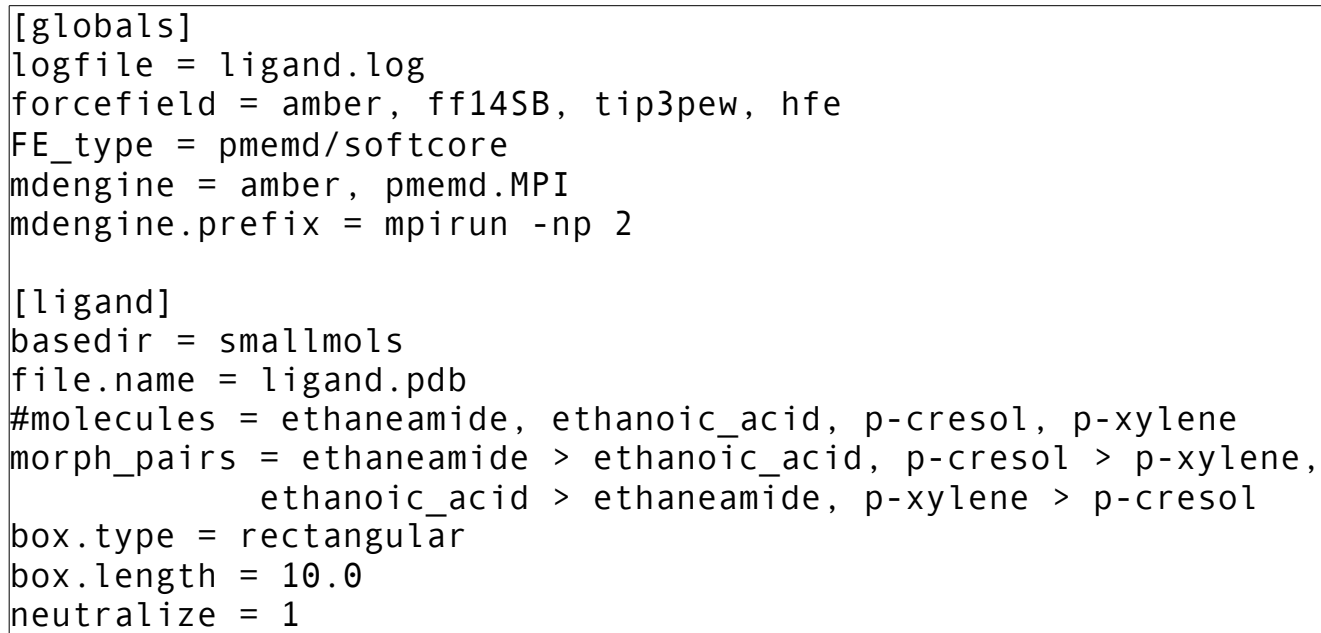

When morph _pairs is not required e.g. for MM-PBSA setup the keyword is replaced with molecules. Start and end states are connected with a '>'. Running FESetup test.in on the command line will create perturbed topology files and template control file. These templates do not prescribe a $\lambda$ path as it is still an open question what would be the best way to do this. In this example, files will be generated for AMBER/pmemd (other formats include Gromacs and Sire).

A more elaborate example is as follows. This provides a complete setup of a complex system and a complex-morph system to be simulated with Gromacs. The complex structure could e.g. come from a previous docking run. Additional force fields can be selected with ff_addons. The input will create simulation boxes for the solvated ligand (and also for vacuum) and the solvated complex. Predefined MD protocols can be run to equilibrate the systems in a set order: minimisation, heating, constant temperature, pressurising (density adjustment) and restraint release steps. 


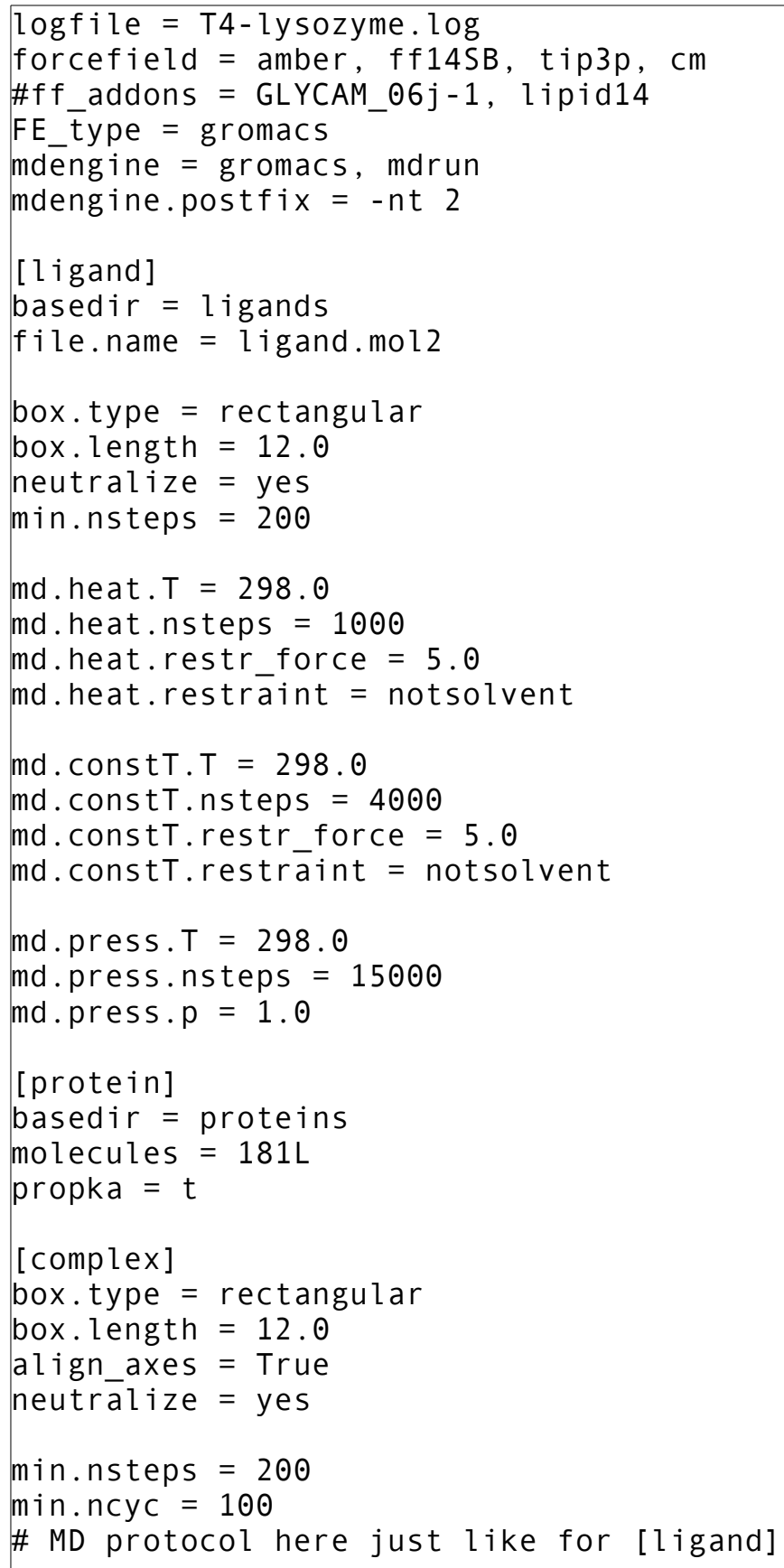


API

The API (application programming interface) allows the user to access the code through their own Python scripts. This allows maximum flexibility and customization. The following simpe example demonstrates the parameterisation of two ligands which are solvated, minimised and heated. The second script picks up on the first and creates all files to carry out a solvation and /or binding free energy simulation. All features as described above in the input examples are accessible through the API.

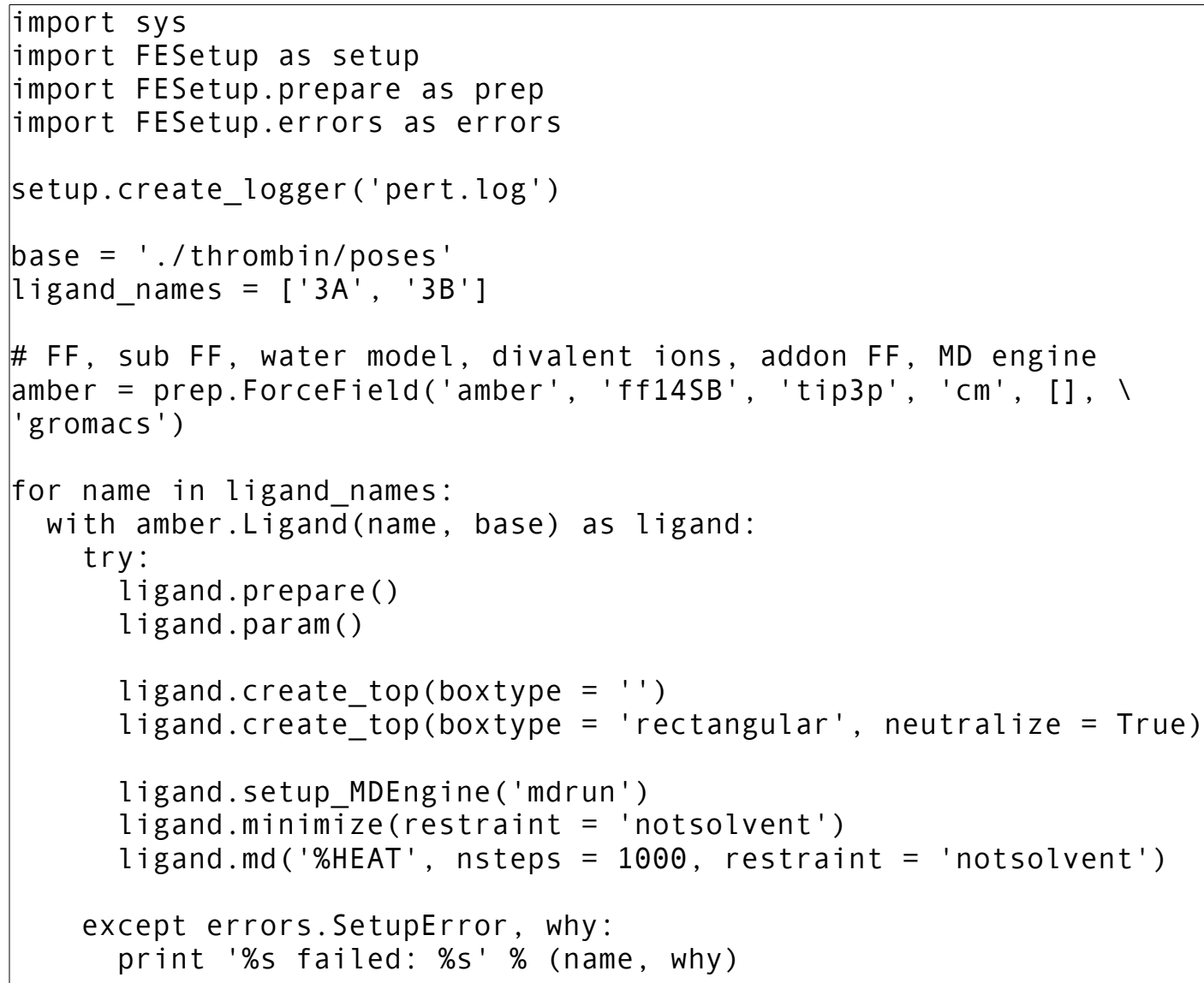




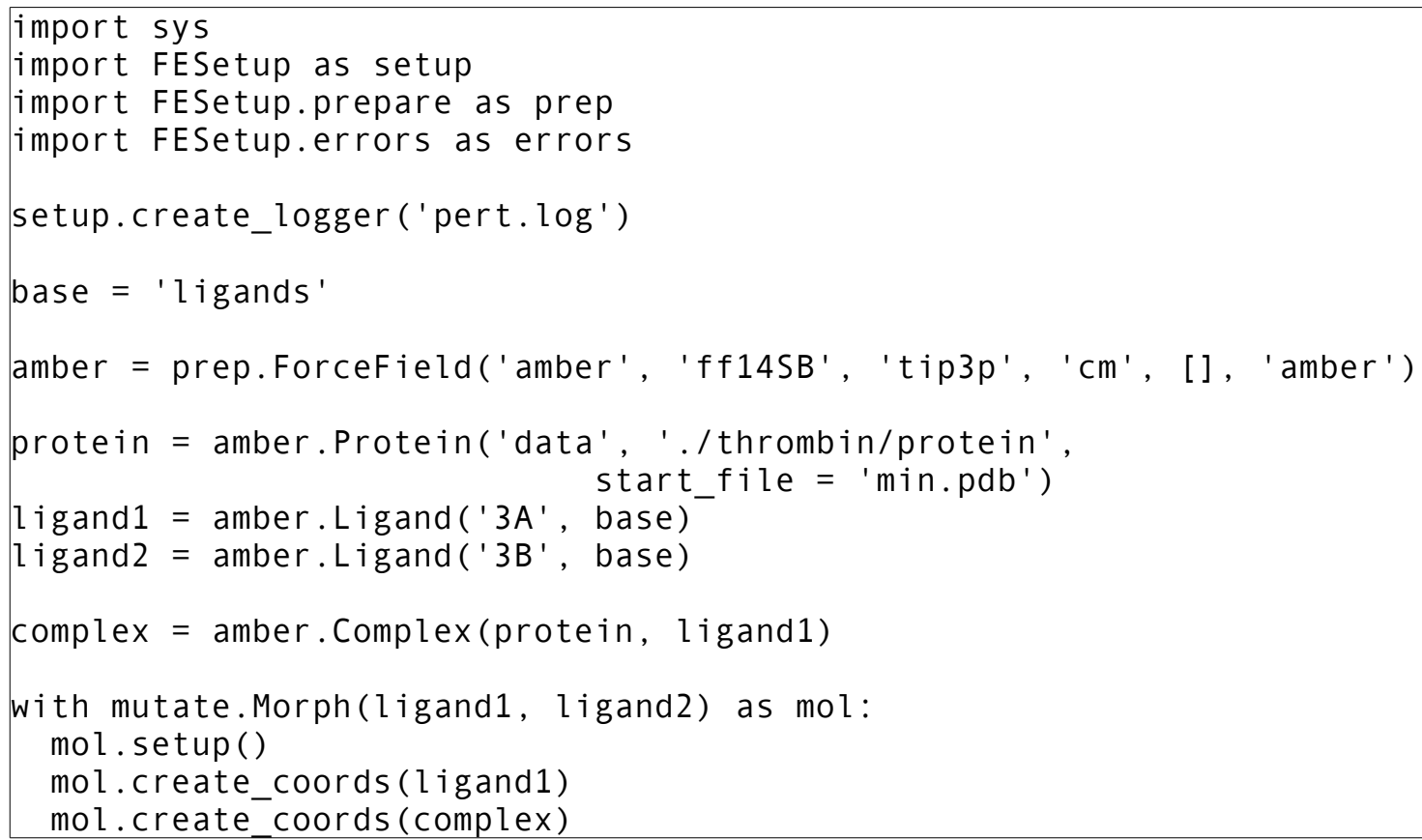

\section{Validation}

Using the first input example (see above) we have created topology and input files for AMBER, GROMACS and Sire, to compute relative hydration free energies. The results are summarized in the following two tables.

Table 1. Free energy changes for the ethaneamide to ethanoic acid transformation in vacuum and in water. All numbers in $\mathrm{kcal} / \mathrm{mol}$.

\begin{tabular}{|c|c|c|c|c|c|c|c|c|}
\hline \multirow{3}{*}{$\begin{array}{l}\text { ethaneamide } \rightarrow \text { ethanoic acid } \\
\text { run1 }\end{array}$} & \multicolumn{4}{|l|}{ AMBER } & \multicolumn{2}{|c|}{ GROMACS } & \multicolumn{2}{|l|}{ Sire } \\
\hline & sol1 & sol2 & vac1 & vac2 & sol & vac & sol & vac \\
\hline & 13.09 & -2.37 & 10.36 & -1.89 & 10.94 & 8.41 & 9.27 & 6.68 \\
\hline run2 & 13.12 & -2.37 & 10.35 & -1.90 & 10.93 & 8.41 & 9.25 & 6.66 \\
\hline run3 & 13.07 & -2.32 & 10.36 & -1.93 & 10.91 & 8.42 & 9.29 & 6.69 \\
\hline 3 -run average & \multicolumn{2}{|c|}{10.74} & \multicolumn{2}{|c|}{8.45} & 10.93 & 8.41 & 9.27 & 6.68 \\
\hline$\Delta \mathbf{G}_{\text {hydr }}$ & \multicolumn{4}{|c|}{2.29} & \multicolumn{2}{|c|}{2.52} & \multicolumn{2}{|c|}{2.59} \\
\hline \multicolumn{9}{|l|}{ ethanoic acid $\rightarrow$ ethaneamide } \\
\hline run1 & -13.07 & 2.35 & -10.36 & 1.93 & -10.84 & -8.32 & -9.24 & -6.68 \\
\hline run2 & -13.11 & 2.31 & -10.36 & 1.89 & -10.83 & -8.32 & -9.28 & -6.69 \\
\hline run3 & -13.09 & 2.44 & -10.36 & 1.93 & -10.84 & -8.33 & -9.29 & -6.68 \\
\hline 3-run average & \multicolumn{2}{|c|}{-10.73} & \multicolumn{2}{|c|}{-8.44} & -10.84 & -8.32 & -9.27 & -6.68 \\
\hline$\Delta \mathbf{G}_{\text {hydr }}$ & \multicolumn{4}{|c|}{-2.29} & \multicolumn{2}{|c|}{-2.52} & \multicolumn{2}{|c|}{-2.59} \\
\hline
\end{tabular}


Table 2. Free energy changes for the p-cresol to p-xylene transformation in vacuum and in water. All numbers in $\mathrm{kcal} / \mathrm{mol}$.

\begin{tabular}{|c|c|c|c|c|c|c|c|c|}
\hline \multirow[b]{2}{*}{$p$-cresol $\rightarrow$ p-xylene } & \multicolumn{4}{|l|}{ AMBER } & \multicolumn{2}{|c|}{ GROMACS } & \multicolumn{2}{|l|}{ Sire } \\
\hline & sol1 & sol2 & vac1 & vac2 & sol & vac & sol & vac \\
\hline run1 & 2.75 & 14.42 & -0.66 & 12.53 & 13.23 & 8.23 & 17.89 & 12.65 \\
\hline run2 & 2.75 & 14.41 & -0.67 & 12.54 & 13.26 & 8.20 & 17.83 & 12.65 \\
\hline run3 & 2.73 & 14.42 & -0.50 & 12.54 & 13.20 & 8.22 & 17.85 & 12.65 \\
\hline 3-run average & \multicolumn{2}{|c|}{17.16} & \multicolumn{2}{|c|}{12.54} & 13.23 & 8.22 & 17.86 & 12.65 \\
\hline$\Delta \mathbf{G}_{\text {hydr }}$ & \multicolumn{4}{|c|}{5.23} & \multicolumn{2}{|c|}{5.01} & \multicolumn{2}{|c|}{5.21} \\
\hline \multicolumn{9}{|l|}{$p$-xylene $\rightarrow p$-cresol } \\
\hline run1 & -2.74 & -14.41 & 0.66 & -12.54 & -13.19 & -8.20 & -17.83 & -12.64 \\
\hline run2 & -2.75 & -14.43 & 0.70 & -12.54 & -13.26 & -8.20 & -17.82 & -12.65 \\
\hline run3 & -2.74 & -14.42 & 0.59 & -12.53 & -13.22 & -8.20 & -17.80 & -12.65 \\
\hline 3-run average & \multicolumn{2}{|c|}{-17.16} & \multicolumn{2}{|c|}{-11.89} & -13.22 & -8.20 & -17.82 & -12.65 \\
\hline$\Delta \mathbf{G}_{\text {hydr }}$ & \multicolumn{4}{|c|}{-5.27} & \multicolumn{2}{|c|}{-5.02} & \multicolumn{2}{|c|}{-5.17} \\
\hline
\end{tabular}

With GROMACS and Sire we have used a 1-step protocol (simultaneous vdW and Coulomb softcore potentials) while with AMBER we had to revert to a 2-step protocol (separation into a Coulomb only transformation and a vdW+bonded transformation). AMBER does support the 1step protocol too but for a yet unknown reason this produces severely wrong results in these cases.

The free energies deviate by up to $0.2 \mathrm{kcal} / \mathrm{mol}$ between MD packages. It has not been established yet to what degree the various MD packages reproduce solvation free energies. We are currently in the process of carrying out a reproducibility study to answer this question.

The results agree well with the absolute free energy results from the FreeSolve project (DOI: $10.1021 /$ ct800409d, results computed with Gromacs), ethaneamide $\rightarrow$ ethanoic acid: $\Delta \mathrm{G}_{\text {hydr }}=2.67$ $\mathrm{kcal} / \mathrm{mol}($ expt. $3.02 \mathrm{kcal} / \mathrm{mol}) ; \mathrm{p}-$ cresol $\rightarrow$ p-xylene: $\Delta \mathrm{G}_{\mathrm{hydr}}=4.69 \mathrm{kcal} / \mathrm{mol}($ expt. 5.33 $\mathrm{kcal} / \mathrm{mol}$ ).Overall, the free energy results show internal consistency between "forward" and "backward" transformations. 
Table 3. Single point energies of morphs computed with GROMACS and Sire. All numbers in $\mathrm{kcal} / \mathrm{mol}$.

\begin{tabular}{|c|c|c|c|c|c|c|c|c|c|}
\hline & & GROMAC & & & & Sire & & & \\
\hline$A$ & B & $A \rightarrow B, \lambda=0$ & $A \rightarrow B, \lambda=1$ & $B \rightarrow A, \lambda=0$ & $B \rightarrow A, \lambda=1$ & $A \rightarrow B, \lambda=0$ & $A \rightarrow B, \lambda=1$ & $B \rightarrow A, \lambda=0$ & $B \rightarrow A, \lambda=1$ \\
\hline benzene & chloro-benzene & 6.09 & 4.19 & 4.19 & 6.09 & 6.09 & 4.19 & 4.19 & 6.09 \\
\hline benzene & cyclopropyl-benzene & 6.19 & 14.23 & 14.23 & 6.19 & 6.19 & 14.23 & 14.23 & 6.19 \\
\hline benzene & ethyl-benzene & 6.14 & 3.10 & 3.10 & 6.14 & 6.14 & 3.10 & 3.10 & 6.14 \\
\hline benzene & methyl-benzene & 6.09 & 2.71 & 2.71 & 6.09 & 6.09 & 2.71 & 2.71 & 6.09 \\
\hline benzene & triazine & 6.09 & -127.22 & 127.22 & 6.09 & 6.09 & -127.22 & 127.22 & 6.09 \\
\hline chloro-benzene & cyclopropyl-benzene & 4.29 & 14.23 & 14.23 & 4.29 & 4.29 & 14.23 & 14.23 & 4.29 \\
\hline cyclopropyl-benzene & triazine & 14.23 & -127.19 & -127.19 & 14.23 & 14.23 & -127.19 & -127.19 & 14.23 \\
\hline ethane & methanol & 1.00 & 3.82 & 3.82 & 1.00 & 1.00 & 3.82 & 3.82 & 1.00 \\
\hline ethane & propane & 1.01 & 1.16 & 1.16 & 1.01 & 1.01 & 1.16 & 1.16 & 1.01 \\
\hline ethane & tert-butane & 1.01 & 0.49 & 0.49 & 1.01 & 1.01 & 0.49 & 0.49 & 1.01 \\
\hline ethyl-benzene & methyl-benzene & 3.10 & 2.72 & 2.72 & 3.10 & 3.10 & 2.72 & 2.72 & 3.10 \\
\hline ethyl-benzene & triazine & 3.10 & -127.22 & 127.22 & 3.10 & 3.10 & -127.22 & 127.22 & 3.10 \\
\hline methane & methanol & 0.09 & 3.81 & 3.81 & 0.09 & 0.09 & 3.81 & 3.81 & 0.09 \\
\hline methane & methyl-benzene & 0.10 & 2.71 & 2.71 & 0.10 & 0.10 & 2.71 & 2.71 & 0.10 \\
\hline methane & propane & 0.11 & 1.16 & 1.16 & 0.11 & 0.11 & 1.16 & 1.16 & 0.11 \\
\hline propane & tert-butane & 1.17 & 0.49 & 0.49 & 1.17 & 1.17 & 0.49 & 0.49 & 1.17 \\
\hline
\end{tabular}

To test code consistency much more rigorously we have also computed single point energies at the lambda end-states, that is for a morph pair $\mathrm{A} / \mathrm{B}$ we have computed the energy for $\mathrm{A} \rightarrow \mathrm{B}$ and $\mathrm{B}$ $\rightarrow$ A at both $\lambda=0.0$ and $\lambda=1.0$. Results are compiled in the Table 3 above. This is a much stricter test because, say, a missing dihedral term can have very little effect on the free energy. Thus bugs in the code may go undetected if we only relied on free energy simulation. We see internal consistency for each code. We also see consistency between Gromacs single point energies and those from Sire.

All structures have been minimised with the conjugate gradient minimizer in Gromacs before energy evaluation. The dihedral terms for dummies have been all set to zero. 